\title{
High Dynamic Range Nanowire Resonators
}

\author{
Juan Molina, Javier E. Escobar, Daniel Ramos, Eduardo Gil-Santos, José J. Ruz, Javier Tamayo, \\ Alvaro San Paulo,* and Montserrat Calleja
}

Cite This: Nano Lett. 2021, 21, 6617-6624

Read Online

\section{ACCESS | Lill Metrics \& More | 回 Article Recommendations | St Supporting Information}

ABSTRACT: Dynamic range quantifies the linear operation regime available in nanomechanical resonators. Nonlinearities dominate the response of flexural beams in the limit of very high aspect ratio and very small diameter, which leads to expectation of low dynamic range for nanowire resonators in general. However, the highest achievable dynamic range for nanowire resonators with practical dimensions remains to be determined. We report dynamic range measurements on singly clamped silicon nanowire resonators reaching remarkably high values of up to $90 \mathrm{~dB}$ obtained with a simple harmonic actuation scheme. We explain these measurements by a comprehensive theoretical examination of dynamic

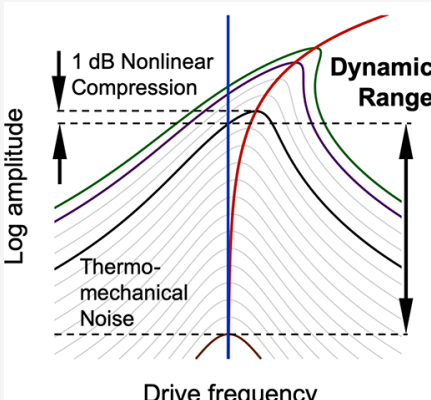

Drive frequency

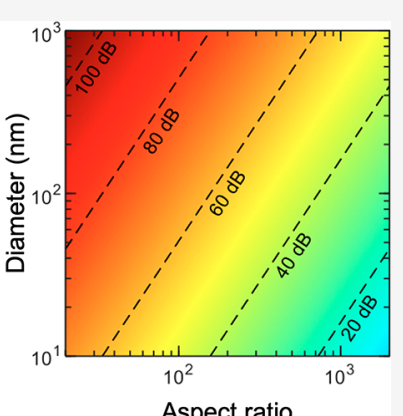

Aspect ratio range in singly clamped flexural beams including the effect of tapering, a usual feature of semiconductor nanowires. Our analysis reveals the nanowire characteristics required for broad linear operation, and given the relationship between dynamic range and mass sensing performance, it also enables analytical determination of mass detection limits, reaching atomic-scale resolution for feasible nanowires.

KEYWORDS: Semiconductor Nanowires, Silicon Nanowires, Nanoelectromechanical Systems (NEMS), Nanomechanical Resonators, Dynamic Range, Nonlinear Dynamics

$\mathrm{N}$ anowire resonators are currently established as one of the most prolific families of nanostructured devices for the realization of nanoelectromechanical systems (NEMS). Their unique physical properties, ranging from optomechanical interactions, ${ }^{1,2}$ resonance degeneration breakage, ${ }^{3-5}$ or mode coupling mechanisms, ${ }^{6,7}$ have enabled a number of highperformance applications as transducers, ${ }^{8-10}$ sensors, ${ }^{3,11-14}$ or microscopy probes. ${ }^{15-17}$ In nanomechanical resonators, nanowires function as flexural beams whose operational regime is determined by the onset of nonlinearity. Standard functionalities require linear schemes based for instance on resonance frequency tracking, which ensures high sensitivity, stable operation, and calibrated measurements. Beyond the nonlinear onset, such schemes cannot be readily used. However, the singular phenomena that emerge in the nonlinear regime enable alternative methods that provide unique functionalities which are otherwise impossible to implement. ${ }^{18}$ In consequence, the practical development of applications based on nanowire resonators requires accurate knowledge about the boundaries between the linear and nonlinear operational regimes. The extent of linear regime available for a particular device is determined by its dynamic range (DR), given by the ratio of the highest linearly driven amplitude to the lowest detectable signal level.

Nanowire resonators, particularly in their single clamp (SC) configuration, have been largely overlooked in terms of DR evaluation. The earliest reports were focused on the double clamp (DC) configuration in the limit of very low diameter and very high aspect ratio. ${ }^{19}$ In such a scenario, nonlinearities appear at low amplitudes and dominate the resonant response, resulting in low values of DR. Although various aspects regarding the onset of nonlinearity in SC beam resonators have been studied, ${ }^{20-26}$ a comprehensive analysis that determines the physical characteristics of nanowire resonators that result in a broad DR is not available. In this work we examine the DR of SC Si nanowire resonators grown by vapor-liquid-solid (VLS) synthesis with a wide range of dimensions. We measure values up to $90 \mathrm{~dB}$ with simple harmonic piezoelectric actuation driving and without using any nonlinear compensation scheme. In order to analyze these results, we develop an analytical theoretical approach to determine the DR of SC flexural beam resonators as a function of their dimensions and geometry, including the effect of cross-section tapering, a common feature in the growth of semiconductor nanowires. $^{27,28}$ The DR predicted by our theoretical analysis for nanowire resonators, which is significantly larger for the SC

Received: May 25, 2021

Revised: July 9, 2021

Published: July 21, 2021 


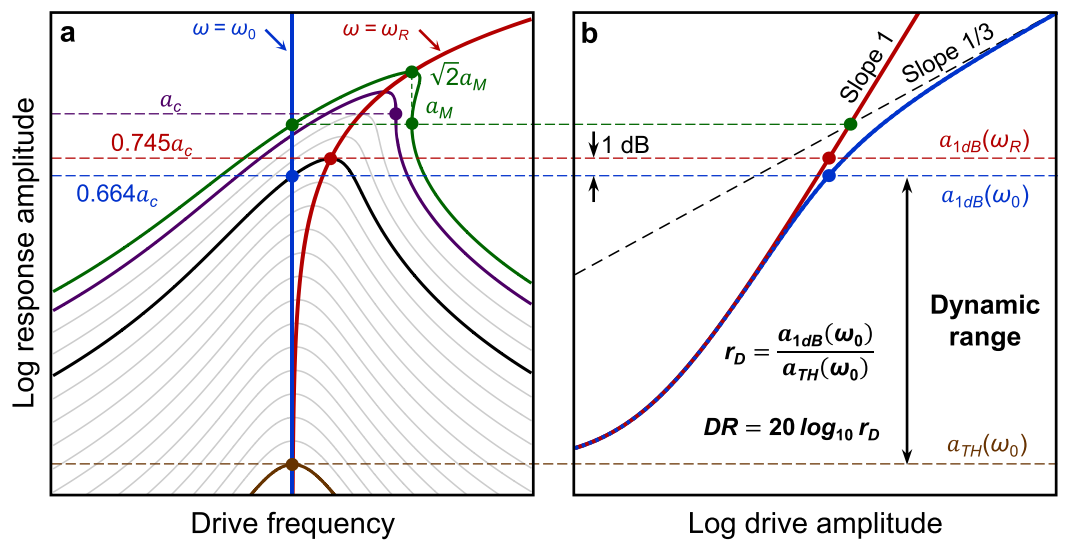

Figure 1. Dynamic range of nanowire resonators. (a) Schematic representation of frequency response curves for varying driving strength, indicating the thermomechanical response curve (brown), the $1 \mathrm{~dB}$ compression curve (black), the critical amplitude curve (purple), and the multivalued resonance frequency threshold curve (green). (b) Schematic representation of the corresponding amplitude vs driving strength curves at $\omega=\omega_{0}$ for the linear (red) and nonlinear (blue) responses, indicating the $1 \mathrm{~dB}$ compression reference points and the resulting spread of the dynamic range (note that if $Q \gg 1$, for a given driving strength the amplitude at $\omega=\omega_{0}$ for the purely linear case matches the amplitude at $\omega=\omega_{\mathrm{R}}$ for the nonlinear response).

configuration than for the DC case, is in very good agreement with our experimental characterization, and it allows expectation of values in the $70-100 \mathrm{~dB}$ range for nanowires with dimensions both feasible and relevant for nanomechanical technology. Finally, we discuss the implications of our results in the context of nanomechanical mass sensing, where DR directly determines the fundamental mass detection limit. Our analytical approach leads to an expression for this limit as a function of the resonator characteristics, which allows predicting an excellent sensing performance for nanowires with ordinary features.

In order to derive an expression for the DR of SC nanowire resonators, we consider that only the fundamental flexural mode is active and that the resonator is driven by an external harmonic force of amplitude $F_{0}$ at frequency $\omega$. Then, the displacement at the free end, $x$, can be described by the following equation of motion: ${ }^{24,29,30}$

$$
\begin{aligned}
m \ddot{x} & +\frac{m \omega_{0}}{Q} \dot{x}+k x+\frac{\beta_{\mathrm{G}}}{L^{2}} x^{3}+\frac{\beta_{\mathrm{I}}}{L^{2}}\left(x \dot{x}^{2}+x^{2} \ddot{x}\right) \\
& =F_{0} \cos (\omega t)
\end{aligned}
$$

where the dot denotes the time derivative and a damping term associated with a finite quality factor $Q$ is included. Consistent with the morphology of most frequently reported nanowire resonators, $^{12}$ we consider a nanowire of length $L$ and hexagonal cross-section with linear tapering described by the coefficient $\alpha_{\mathrm{T}}=1-D_{\text {tip }} / D_{0}$, where $D_{\text {tip }}$ and $D_{0}$ are the diameter values at the tip and base, respectively. The effective mass $m$, effective spring constant $k$, and natural frequency $\omega_{0}$ are related by $\omega_{0}{ }^{2}=k / m$. These parameters, together with the geometrical $\beta_{\mathrm{G}}$ and inertial $\beta_{\mathrm{I}}$ nonlinear coefficients, are determined by the nanowire geometry (cross-section area at the base $S_{0}$, second moment of area at the base $I_{0}$, and tapering coefficient $\alpha_{\mathrm{T}}$ ), material properties (density $\rho$ and Young's modulus $E$ ), and flexural mode shape as given by EulerBernoulli theory ${ }^{31}$ (see Supporting Information).

Introducing a global nonlinear coefficient as $\alpha_{\mathrm{NL}}=\beta_{\mathrm{G}} / k-$ $2 \beta_{\mathrm{I}} /(3 m)$ leads to a compact expression for the frequency response of the nanowire. In the case of large aspect ratio $(L \gg$ $\left.D_{0}\right), \alpha_{\mathrm{NL}}$ is positive for the fundamental mode, ${ }^{25,26}$ and the frequency response can be written as

$$
a^{2}(\omega)=\frac{a_{0}{ }^{2}}{\left[Q\left(\frac{\omega^{2}}{\omega_{0}{ }^{2}}-1\right)-\frac{a^{2}(\omega)}{a_{\mathrm{M}}{ }^{2}}\right]^{2}+\frac{\omega^{2}}{\omega_{0}{ }^{2}}}
$$

where $a$ represents the beam oscillation amplitude and we define $a_{0}=Q F_{0} / k$ and $a_{\mathrm{M}}=2 L / \sqrt{3 Q \alpha_{\mathrm{NL}}}$. Thus, $a_{0}$ represents the beam oscillation amplitude at the natural frequency in the linear regime and $a_{\mathrm{M}}$ is a characteristic magnitude of the nonlinear onset. If $\alpha_{\mathrm{NL}}=0$, eq 2 reduces to the Lorentzian response of a linear resonator, and the resonance frequency is $\omega_{\mathrm{R}} \approx \omega_{0}$ for $Q \gg 1$; if $\alpha_{\mathrm{NL}}>0$, the resonance frequency is given by $\omega_{\mathrm{R}}^{2} \approx \omega_{0}^{2}\left[1+a_{0}^{2} /\left(Q a_{\mathrm{M}}^{2}\right)\right]$. The parameter $a_{\mathrm{M}}$ provides a measure of the ultimate limit of the linear regime (Figure 1a): when the driving force is large enough to raise the amplitude at the natural frequency to $a\left(\omega_{0}\right)=a_{\mathrm{M}}$, then the resonance frequency becomes multivalued so that $a\left(\omega_{\mathrm{R}}\right)_{\mathrm{I}}=\sqrt{2} a_{\mathrm{M}}$ (resonance peak amplitude) and $a\left(\omega_{\mathrm{R}}\right)_{\mathrm{II}}=$ $a_{\mathrm{M}}$ (bifurcation point). Thus, the driving force for which $a\left(\omega_{0}\right)$ $=a_{\mathrm{M}}$ represents the threshold for a multivalued resonance frequency, above which standard resonance frequency tracking schemes used in the linear regime become absolutely impracticable.

A practical reference to define the onset of nonlinearity is the $1 \mathrm{~dB}$ compression point, ${ }^{32}$ referred to as the point where the oscillation amplitude at the natural frequency $a_{1 \mathrm{~dB}}\left(\omega_{0}\right)$ is 1 $\mathrm{dB}$ lower than the amplitude that would result from a purely linear response for the same driving force (Figure $1 \mathrm{~b}$ ). The 1 $\mathrm{dB}$ compression point can be related to the critical amplitude $a_{\mathrm{C}}$, defined as the amplitude at the bifurcation point in the frequency response curve corresponding to a driving force for which the curve begins to be multivalued (Figure 1a). This relation results in $a_{1 \mathrm{~dB}}\left(\omega_{0}\right) \simeq 0.664 a_{\mathrm{C}}$, although the relation of $a_{\mathrm{C}}$ with the amplitude at resonance $a_{1 \mathrm{~dB}}\left(\omega_{\mathrm{R}}\right) \simeq 0.745 a_{\mathrm{C}}$ has been typically considered in previous works ${ }^{19,33,34}$ (see Supporting Information). A more straightforward approach to determine $a_{1 \mathrm{~dB}}\left(\omega_{0}\right)$ can be followed by using eq 2 for writing the $1 \mathrm{~dB}$ compression condition as $a_{0} / a_{1 \mathrm{~dB}}\left(\omega_{0}\right)=$ $10^{1 / 20}$, which leads to

$$
a_{1 \mathrm{~dB}}\left(\omega_{0}\right)=\left(10^{1 / 10}-1\right)^{1 / 4} a_{\mathrm{M}} \simeq 0.713 a_{\mathrm{M}}
$$



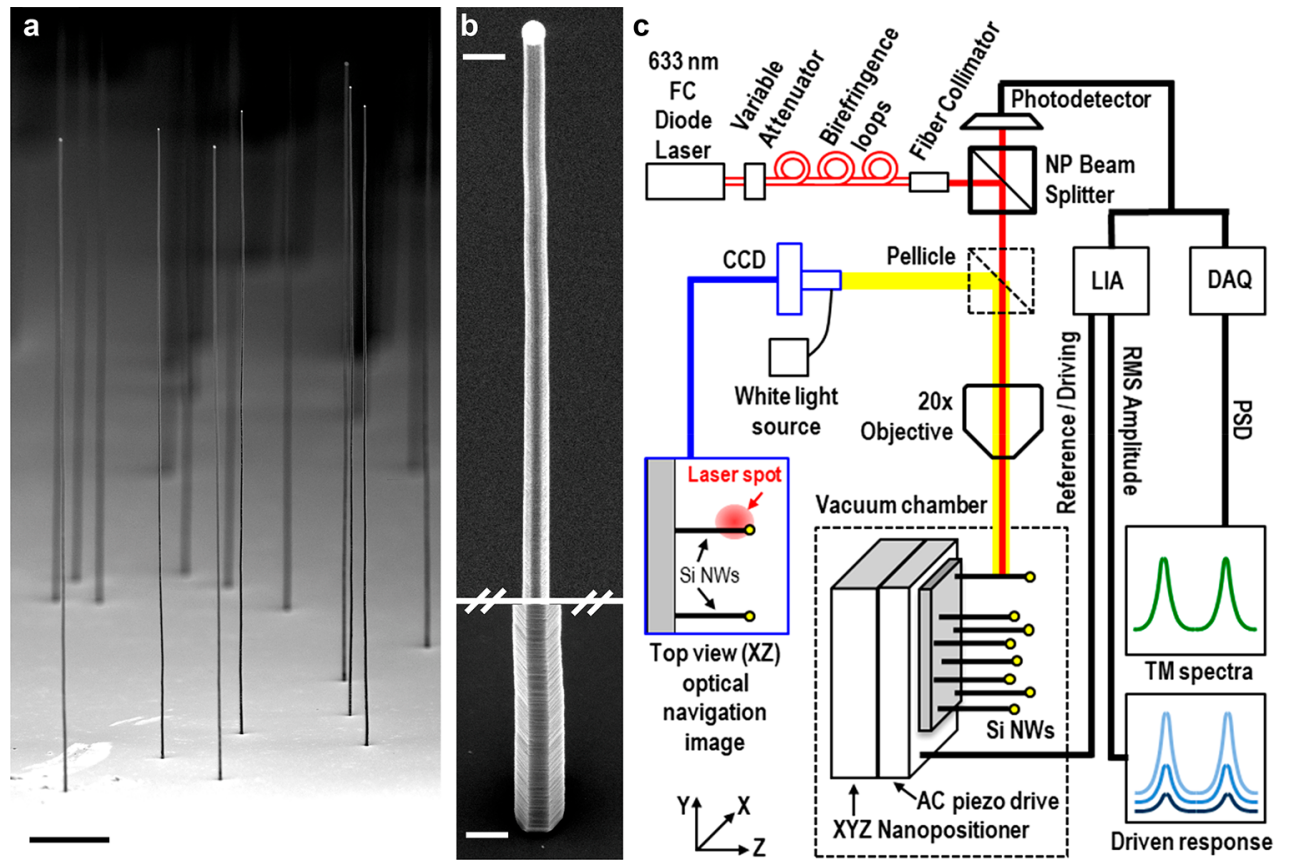

Figure 2. Experimental system and measurement setup. (a) Scanning electron microscopy image of a representative sample of the Si nanowires used in this work (scale bar $5 \mu \mathrm{m}$ ). (b) Details of the cross-section geometry along a nanowire length, which evolves from hexagonal at the base (lower image section) and most nanowire length (>50\%) to polyhedral near the tip (upper image section; scale bar $300 \mathrm{~nm}$ in both sections). (c) Schematic depiction of the measurement setup, based on backscattered light modulation transduction. A diode laser $(\lambda=633 \mathrm{~nm})$ is focused by a $0.42 \mathrm{NA}$ objective on the $\mathrm{Si}$ nanowires placed in a high-vacuum chamber $\left(\sim 10^{-5} \mathrm{mbar}\right)$. The incident laser beam is linearly polarized along the longitudinal axis of the nanowires, and its optical power ranges from 72 to $216 \mu \mathrm{W}$. The backscattered beam is collected by an amplified photodetector whose output signal is processed either by a digital acquisition (DAQ) board or by a lock-in amplifier (LIA).

The lower limit for determining the DR is given by the noise floor of the amplitude signal. Here we model the intrinsic DR of a beam, so we consider thermomechanical noise as the dominant noise source. Thus, the corresponding spectral density at resonance is

$$
S_{x}^{T h}\left(\omega_{0}\right)=\frac{4 k_{\mathrm{B}} T Q}{m \omega_{0}^{3}}
$$

where $k_{\mathrm{B}}$ is the Boltzmann's constant and $T$ is the temperature.

We define the dynamic ratio $r_{\mathrm{D}}$ as the ratio of the amplitude at the onset of nonlinearity ( $1 \mathrm{~dB}$ compression point) to the lowest measurable amplitude (thermomechanical spectral density integrated for the measurement bandwidth $\Delta f$ ):

$$
r_{\mathrm{D}}=\frac{a_{1 \mathrm{~dB}}\left(\omega_{0}\right)}{\sqrt{2 S_{x}^{\mathrm{Th}} \Delta f}} \simeq 0.291 \sqrt{\frac{L^{2} m \omega_{0}^{3}}{k_{\mathrm{B}} T Q^{2} \Delta f\left|\alpha_{\mathrm{NL}}\right|}}
$$

We preserve the term dynamic range (DR) for the ratio $r_{\mathrm{D}}$ expressed in $\mathrm{dB}$ so that $\mathrm{DR}=20 \log _{10} r_{\mathrm{D}}$. The exact calculation of the DR from eq 5 involves the computation of $m, \omega_{0}$, and $\alpha_{\mathrm{NL}}$ as a function of the beam geometry, material properties, and mode shape, which requires numerical methods. Note that this calculation requires considering the mode shape of a tapered nanowire, as computed in a previous work. ${ }^{28}$ However, an excellent analytical approximation can be obtained by separating the contribution of tapering in a function $h\left(\alpha_{\mathrm{T}}\right)$. Then, introducing the corresponding expressions of $S_{0}$ and $I_{0}$ for a hexagonal cross-section, the DR of nanowire resonators can be calculated from

$$
r_{\mathrm{D}} \simeq 0.371 h\left(\alpha_{\mathrm{T}}\right) D_{0}\left(\frac{D_{0}}{L}\right)^{3 / 2} \sqrt{\frac{E^{3 / 2}}{Q^{2} k_{\mathrm{B}} T \Delta f \sqrt{\rho}}}
$$

The function $h\left(\alpha_{\mathrm{T}}\right)$ can be approximated by $h\left(\alpha_{\mathrm{T}}\right)=\sum_{i} c_{i} \alpha_{\mathrm{T}}^{i}$, and the coefficients $c_{i}$ can be obtained by fitting $h\left(\alpha_{\mathrm{T}}\right)$ to numerical calculations of $r_{\mathrm{D}}$ from eq 5 (see Supporting Information). For $\alpha_{\mathrm{T}}<0.9, h\left(\alpha_{\mathrm{T}}\right)$ can be simplified to $h\left(\alpha_{\mathrm{T}}\right) \simeq$ $1-0.677 \alpha_{\mathrm{T}}$ so that for nanowires with uniform section, $h(0)$ $\simeq 1$.

Equation 6 points out some important differences between SC and DC beams, particularly regarding the dependence on the aspect ratio $L / D_{0}$. This expression is notably analogous to the ones previously derived for the DC configuration. ${ }^{19}$ However, the SC case shows a weaker dependence on the aspect ratio, $r_{\mathrm{D}} \propto D_{0}\left(L / D_{0}\right)^{-3 / 2}$, as compared to the DC configuration, where $r_{\mathrm{D}} \propto D_{0}\left(L / D_{0}\right)^{-5 / 2}$ so that DR does not degrade for increasing aspect ratio as fast as for the DC case. The quantitative relation between the $\mathrm{DR}$ of $\mathrm{SC}$ and $\mathrm{DC}$ nanowires with equal characteristics is approximated by $r_{\mathrm{D}}(\mathrm{SC}) / r_{\mathrm{D}}(\mathrm{DC}) \simeq 0.233 L / D_{0}$. Therefore, for approximately $L / D_{0}>50$, the DR of SC nanowires is more than $20 \mathrm{~dB}$ larger than that of equivalent DC nanowires. Similar results are obtained for beams with circular and rectangular cross-section (see Supporting Information). The physical origin of this disparity lies in the different mechanisms that dominate nonlinearities for each clamping configuration. In DC beams, a relatively small beam deflection necessarily implies that the beam stretches so that bending-induced tension dominates. In SC beams, bending produces negligible stretching, and nonlinearity is provided by bending-induced curvature. This is a lower magnitude effect so that SC beams reach the 

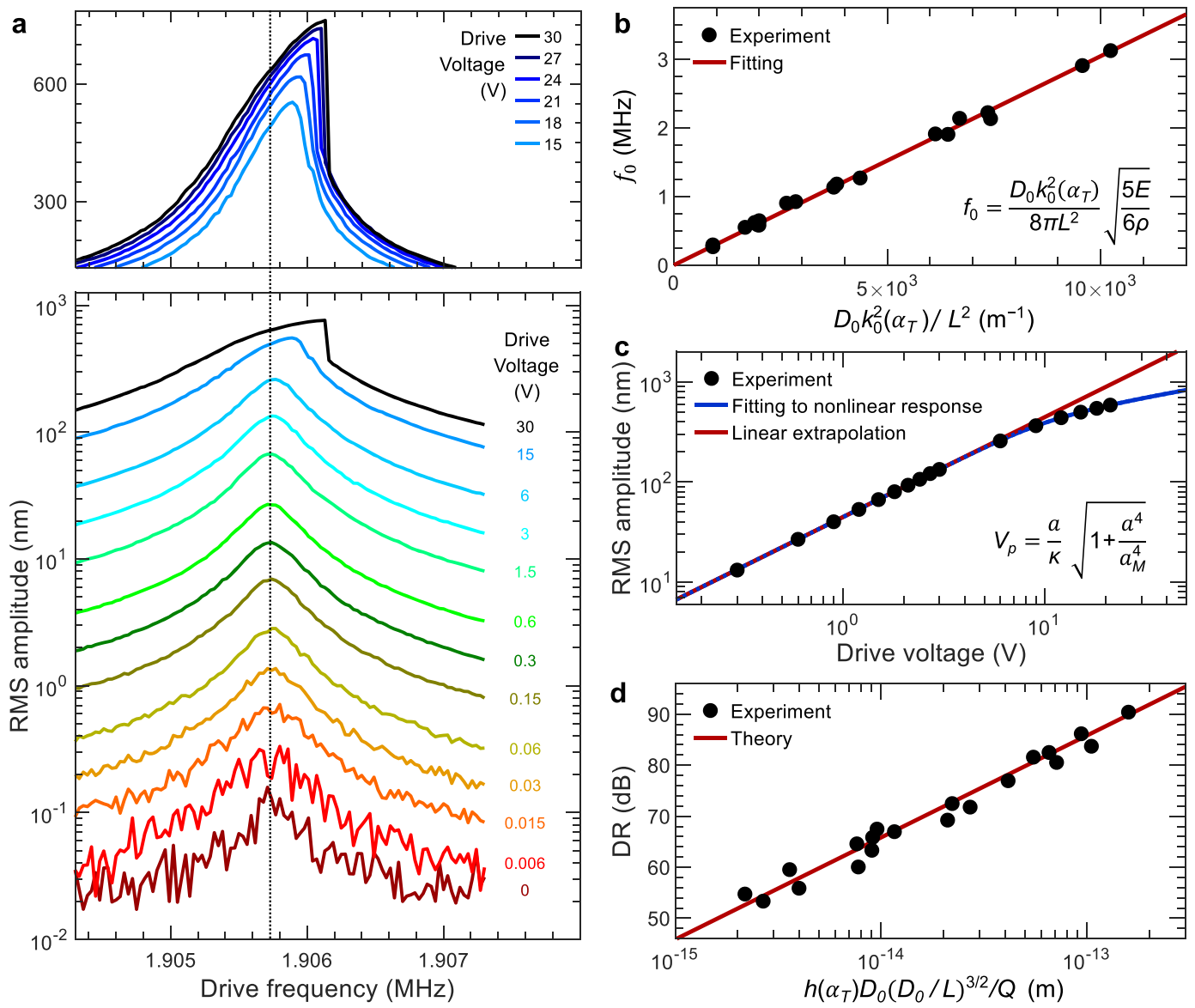

Figure 3. Dynamic range measurements and experiment-theory comparison. (a) Representative example of the measured frequency response curves for varying driving strength for a nanowire with $f_{0}=1905.7 \mathrm{kHz}$ (dotted line) and $Q=4867$. The lower section shows the full set of curves from the thermomechanical response to the nonlinear regime. The upper section shows a subset of curves around the nonlinear onset $(\Delta f=14.82$ $\mathrm{Hz}$ ). (b) Measurements and fitting of the natural frequencies of all nanowires tested in this work, from which a Young's modulus $E=164 \pm 6 \mathrm{GPa}$ is obtained. (c) Example of a measurement of the amplitude at the natural frequency for varying driving strength and fitting to the theoretical expression derived in the main text $(\Delta f=1.098 \mathrm{~Hz})$. (d) Experimental results and theoretical calculation of the dynamic range for all nanowires under test.

nonlinear onset at higher amplitudes than equivalent DC beams.

In order to characterize the DR of nanowire resonators, we have performed experimental measurements on $\mathrm{Si}$ nanowires vertically grown on flat $\mathrm{Si}$ substrates by the VLS mechanism (see Supporting Information), resulting in SC beam resonators where the clamp is defined by the epitaxial union of the nanowire base to the substrate. ${ }^{35}$ Figure $2 \mathrm{a}$ shows a representative scanning electron microscopy (SEM) image of the $\mathrm{Si}$ nanowires under test. The nanowires have lengths in the range of $10-50 \mu \mathrm{m}$, base diameters of $125-425 \mathrm{~nm}$, and tapering coefficients of $0.1-0.9$. The resulting aspect ratio $L /$ $D_{0}$ is in the range 30-180. A precise measurement of nanowire dimensions is obtained from high resolution SEM images. ${ }^{10}$ The cross-section at the base is hexagonal with an approximately regular geometry, but the sidewall facets evolve along the nanowire length so that near the tip the cross-section becomes polyhedral (Figure 2b). The gold catalyst nanoparticle used for the VLS synthesis generates a gold-silicon oxide core-shell nanostructure that remains at the tip of the nanowire. $^{36}$ The nanowires are grown on plain $\mathrm{Si}(111)$ substrates without any kind of prefabricated structure.

The frequency response of the nanowires is measured in high vacuum by the optical transduction setup depicted in
Figure 2c (see Supporting Information). The nanowires are driven by a piezoelectric actuator placed under the substrate, and their vibrations are measured by collecting the backscattered light resulting from the incidence of a focused laser beam. ${ }^{10}$ We use a laser beam incidence perpendicular to the nanowires in order to match the most commonly extended configuration in applications of nanowire resonators. ${ }^{12}$ The transduction mechanism relies on vibration-induced light scattering modulation (see Supporting Information). Such mechanism provides a wide linear transduction range that ensures undistorted oscillation amplitude measurements, whereas interferometric approaches can be more limited regarding this issue. ${ }^{25}$ For every single nanowire tested in this work we have verified that the thermomechanical amplitude at the natural frequency is above the transduction detection limit and that the amplitude at the $1 \mathrm{~dB}$ compression point is within the transduction linear range (see Supporting Information).

The results of the characterization of the DR of the $\mathrm{Si}$ nanowires under analysis are shown in Figure 3. In Figure 3a we present an example of a set of resonance curves acquired with varying driving strength, revealing the nanowire response from the thermomechanical spectrum to slightly above the onset of nonlinearity. This example corresponds to the upper 
Table 1. Set of Si Nanowire Resonators Tested in This Work

\begin{tabular}{|c|c|c|c|c|c|c|c|c|c|c|c|}
\hline no. & $L(\mu \mathrm{m})$ & $D_{0}(\mathrm{~nm})$ & $L / D_{0}$ & $a_{\mathrm{T}}$ & $m(\mathrm{fg})$ & $Q$ & $f_{\exp }(\mathrm{kHz})$ & $f_{\text {theo }}(\mathrm{kHz})$ & $\mathrm{DR}_{\exp }(\mathrm{dB})$ & $\mathrm{DR}_{\text {theo }}(\mathrm{dB})$ & $\delta m(\mathrm{zg})$ \\
\hline 1 & 43.2 & 241 & 179 & 0.89 & 46 & 15116 & 294 & 278 & 53 & 54 & 6.61 \\
\hline 2 & 44.2 & 256 & 173 & 0.88 & 56 & 20947 & 280 & 278 & 55 & 53 & 4.91 \\
\hline 3 & 43.7 & 276 & 158 & 0.81 & 100 & 15717 & 267 & 278 & 56 & 58 & 10.27 \\
\hline 4 & 25.9 & 199 & 130 & 0.72 & 50 & 19110 & 550 & 508 & 60 & 57 & 2.79 \\
\hline 5 & 17.6 & 212 & 83 & 0.34 & 145 & 27860 & 925 & 869 & 60 & 64 & 5.17 \\
\hline 6 & 25.7 & 237 & 108 & 0.69 & 81 & 12411 & 589 & 596 & 63 & 65 & 4.46 \\
\hline 7 & 25.1 & 219 & 115 & 0.73 & 55 & 11790 & 582 & 607 & 65 & 63 & 2.75 \\
\hline 8 & 27.0 & 294 & 92 & 0.52 & 255 & 23921 & 621 & 576 & 66 & 65 & 5.44 \\
\hline 9 & 27.1 & 326 & 83 & 0.46 & 378 & 25566 & 646 & 608 & 67 & 67 & 6.63 \\
\hline 10 & 17.5 & 190 & 92 & 0.38 & 105 & 16724 & 903 & 804 & 67 & 65 & 2.65 \\
\hline 11 & 18.1 & 309 & 58 & 0.28 & 369 & 26806 & 1181 & 1162 & 69 & 72 & 4.75 \\
\hline 12 & 19.3 & 347 & 56 & 0.27 & 502 & 25265 & 1135 & 1141 & 72 & 74 & 5.11 \\
\hline 13 & 10.3 & 145 & 71 & 0.46 & 29 & 7557 & 1912 & 1866 & 72 & 73 & 0.90 \\
\hline 14 & 10.3 & 192 & 53 & 0.29 & 79 & 9567 & 2222 & 2240 & 77 & 78 & 1.17 \\
\hline 15 & 11.6 & 338 & 34 & 0.17 & 357 & 20909 & 2909 & 2915 & 81 & 83 & 1.60 \\
\hline 16 & 19.5 & 422 & 46 & 0.24 & 802 & 20365 & 1270 & 1329 & 82 & 81 & 3.28 \\
\hline 17 & 10.8 & 183 & 59 & 0.31 & 71 & 4867 & 1906 & 1956 & 83 & 82 & 1.10 \\
\hline 18 & 10.7 & 194 & 55 & 0.24 & 94 & 3789 & 2139 & 2039 & 84 & 86 & 1.61 \\
\hline 19 & 10.5 & 203 & 52 & 0.29 & 91 & 4701 & 2134 & 2260 & 86 & 85 & 0.95 \\
\hline 20 & 11.0 & 326 & 34 & 0.18 & 308 & 9097 & 3127 & 3116 & 90 & 90 & 1.02 \\
\hline
\end{tabular}
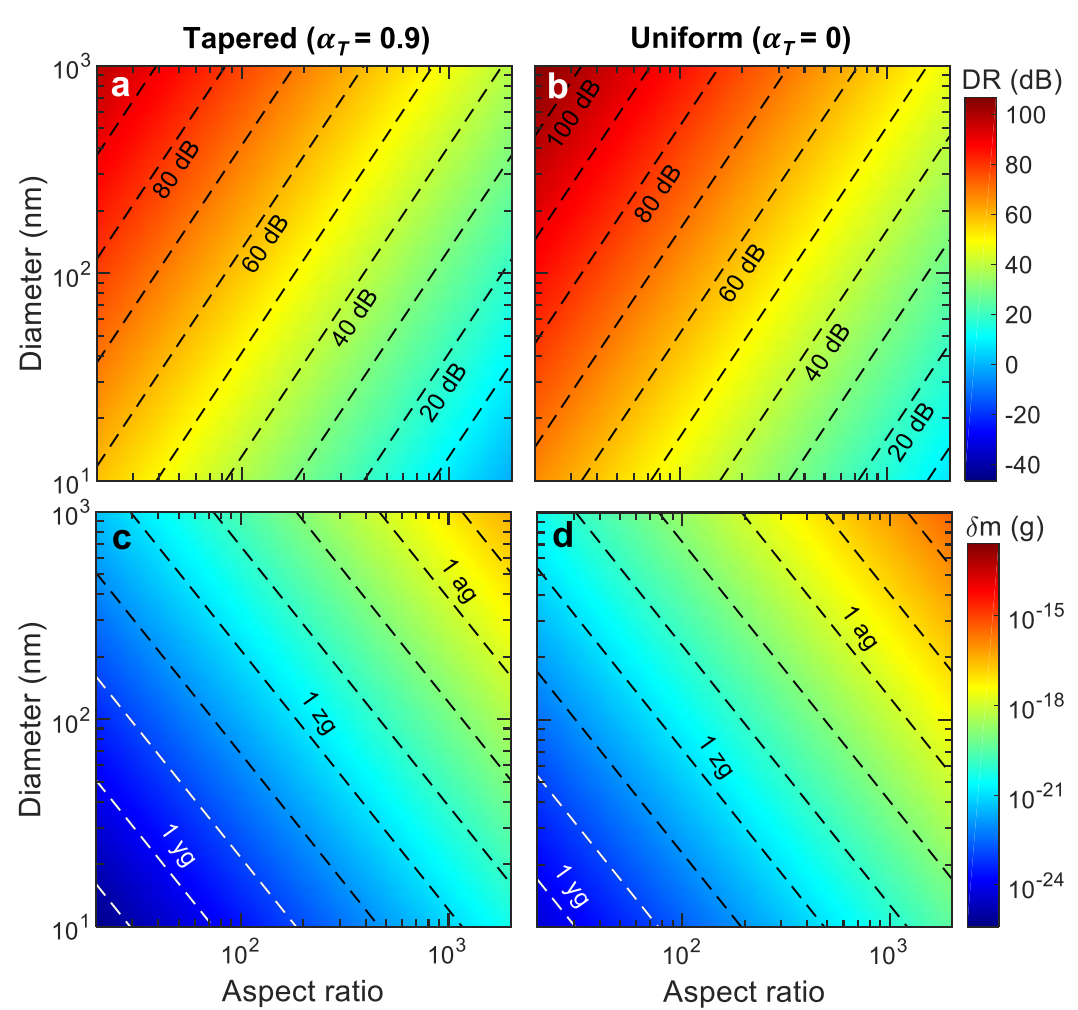

Figure 4. Theoretical calculations of dynamic range and fundamental mass detection limit for singly clamped Si nanowire resonators. (a) Dynamic range for tapered nanowires $\left(a_{\mathrm{T}}=0.9\right)$. (b) Dynamic range for uniform nanowires $\left(a_{\mathrm{T}}=0\right)$. (c) Fundamental mass detection limit for tapered nanowires $\left(a_{\mathrm{T}}=0.9\right)$. (d) Fundamental mass detection limit for uniform nanowires $\left(a_{\mathrm{T}}=0\right)$.

resonance peak of the fundamental mode doublet, ${ }^{3}$ acquired by upward frequency sweeps on a Si nanowire with a length of $10.8 \mu \mathrm{m}$, a base diameter of $183 \mathrm{~nm}$, and a tapering coefficient of 0.3 . Thermomechanical calibration has been used to convert the measured signal into length units. ${ }^{37}$ These measurements allow determination of the natural frequency and quality factor of the nanowires, which for this example result in $1.906 \mathrm{MHz}$ and 4867, respectively. Figure $3 \mathrm{~b}$ shows a plot of the fundamental natural frequency of the 20 nanowires presented in this study. The natural frequencies are in the range of $0.2-$ $3.5 \mathrm{MHz}$ and the quality factors in the range of 3000-30000. The experimental frequency data are fitted to the equation derived for a tapered hexagonal cross-section in which the tapering effect is contained in a polynomic function $k_{0}\left(\alpha_{\mathrm{T}}\right){ }^{28}$ The fitting results in a value of Young's modulus $E=164 \pm 6$ $\mathrm{GPa}$, in good agreement with the bulk value for $\mathrm{Si}(111)$ of 187 $\mathrm{GPa}$ as expected for $\mathrm{Si}$ nanowires in this range of diameters assuming the bulk Si density $\rho=2329 \mathrm{~kg} / \mathrm{m}^{3}{ }^{38}$ The small 
discrepancy is attributed to the varying geometry of the nanowire cross-section along its length, to the presence of sidewall sawtooth faceting, and to the core-shell nanostructure at the tip, which may affect the effective mass and spring constant.

Precise measurements of DR are performed as exemplified in Figure 3c. We determine the amplitude at the $1 \mathrm{~dB}$ compression point by a series of measurements of the amplitude at the natural frequency for varying actuator driving voltage $V_{\mathrm{p}}$. We fit such measurements to the theoretical expression that can be derived by making $\omega=\omega_{0}$ in eq 2 :

$$
V_{\mathrm{p}}=\frac{a}{\kappa} \sqrt{1+\frac{a^{4}}{a_{\mathrm{M}}^{4}}}
$$

where we assume that the amplitude in the linear regime is proportional to $V_{\mathrm{p}}$ so that $a_{0}=\kappa V_{\mathrm{p}}$. From such fitting we extract $\kappa$ and $a_{\mathrm{M}}$, and the latter is used to calculate $a_{1 \mathrm{~dB}}\left(\omega_{0}\right)$ by using eq 3 . The thermomechanical response amplitude $a_{\mathrm{TH}}\left(\omega_{0}\right)$ is measured by setting $V_{\mathrm{p}}=0$, and then the experimental DR is obtained from $r_{\mathrm{D}}=a_{1 \mathrm{~dB}}\left(\omega_{0}\right) / a_{\mathrm{TH}}\left(\omega_{0}\right)$. Figure $3 \mathrm{~d}$ presents all the measured values (dots) of DR vs the factor that combines all the experimentally measured parameters that determine the DR according to eq 6 . We also directly plot (line) the theoretical behavior expected from eq 6 by using the value of $E$ determined from the fitting of the resonance frequencies and considering the experimental conditions $(\Delta f=1.098 \mathrm{~Hz}, T=325 \mathrm{~K})$. Table 1 summarizes all experimental and theoretical values for the DR and other relevant parameters for the whole set of nanowires analyzed in this work. The very good agreement between measurements and theory supports our analytical approach and the compliance of nanowire dynamics with Euler-Bernoulli theory in their full dynamic range.

The highest DR measured for the set of nanowires under test in this work is $90 \mathrm{~dB}$. This value is comparable to the highest DR values reported for other nanomechanical resonators based on either nonlinearity compensated DC beams, ${ }^{39-41}$ SC rectangular beams with lower aspect ratio, ${ }^{42}$ or harmonically driven atomically thin membranes, ${ }^{33,34}$ which have been recently reported to naturally provide large DR values. Moreover, the theoretical analysis presented here allows us to estimate that even higher DR values can be achieved with nanowire resonators with feasible dimensions. Figure 4 displays a calculation of the DR of Si nanowires as a function of their aspect ratio and diameter. For this calculation we consider $Q=10000, \Delta f=1.098 \mathrm{~Hz}, E=164 \mathrm{GPa}, T=325 \mathrm{~K}$, and $\rho=2329 \mathrm{~kg} / \mathrm{m}^{3}$. The graphs in Figure $4 \mathrm{a}$ and Figure $4 \mathrm{~b}$ allow us to compare the cases of a SC nanowire with $\alpha_{\mathrm{T}}=0.9$ (highly tapered) and $\alpha_{\mathrm{T}}=0$ (uniform), respectively. From the approximation used for $h\left(\alpha_{\mathrm{T}}\right)$, it can be estimated that a nanowire with uniform cross-section provides around $8.2 \mathrm{~dB}$ of DR above a tapered nanowire with $\alpha_{\mathrm{T}}=0.9$ and the same dimensions and quality factor. Figure $4 \mathrm{~b}$ shows that values of DR above $70 \mathrm{~dB}$ and up to the $100 \mathrm{~dB}$ level can be reached in uniform Si nanowires when combining an aspect ratio below 100 and a diameter above $100 \mathrm{~nm}$. This range of dimensions is consistent with values typically found in reports of Si nanowire resonator applications, ${ }^{12}$ which point out the feasibility and relevance of nanowire resonators with dimensions resulting in high dynamic range.

SC beam resonators have been extensively used as excellent platforms for ultrasensitive mass detection, ${ }^{4-45}$ and $\mathrm{Si}$ nanowires in particular provide various unique functional features. $^{1,3,28,46}$ The fundamental mass detection limit $\delta m$ in frequency tracking measurement with a phase lock loop readout at constant mean square amplitude is determined by $\mathrm{DR}$ as $\delta m=m /\left(Q r_{\mathrm{D}}\right),{ }^{47}$ which assumes (1) driving at the onset of nonlinearity, (2) frequency stability limited by thermomechanical fluctuations, and (3) added mass placed at the point of maximum displacement. Although mass sensing with beam resonators is feasible in the nonlinear regime ${ }^{48}$ and frequency stability may not be limited by thermomechanical fluctuations but other noise sources, ${ }^{42}$ it is useful to refer to $\delta m$ as an ultimate limit that allows to compare different devices. $^{26,34}$ Our derivation of the DR for nanowire resonators leads to

$$
\delta m \simeq 0.438 j\left(\alpha_{\mathrm{T}}\right) D_{0}{ }^{2}\left(\frac{L}{D_{0}}\right)^{5 / 2} \sqrt{\frac{k_{\mathrm{B}} T \Delta f \rho^{5 / 2}}{E^{3 / 2}}}
$$

where $j\left(\alpha_{\mathrm{T}}\right)$ contains the tapering effect, and it can be approximated by $j\left(\alpha_{\mathrm{T}}\right) \simeq 1-1.171 \alpha_{\mathrm{T}}+0.200 \alpha_{\mathrm{T}}{ }^{2}$ (see Supporting Information). Table 1 presents the values of $\delta m$ obtained for the nanowire resonators under test, which lay in the range of a few zeptograms. Figure $4 \mathrm{c}$ and Figure $4 \mathrm{~d}$ present the calculations of $\delta m$ for SC Si nanowires with the same parameters as in Figure $4 \mathrm{a}$ and Figure $4 \mathrm{~b}$, respectively. Tapered nanowires approach $1 \mathrm{yg}$ for aspect ratio below 100 and diameter below $100 \mathrm{~nm}$. Remarkably, $\delta m$ is in general much lower for tapered nanowires than for uniform cross-sections. By evaluating $j\left(\alpha_{\mathrm{T}}\right)$, we find $\delta m\left(\alpha_{\mathrm{T}}=0.9\right) / \delta m\left(\alpha_{\mathrm{T}}=0\right) \simeq 0.11$, which implies that $\delta m$ for tapered nanowires is around 1 order of magnitude lower than that of uniform cross-section nanowires with the same length and diameter at the base. Although tapering results in lower DR, the effective mass contribution, significantly reduced with tapering, dominates in $\delta m$. Regarding the $Q$ factor, in spite of improving resonance frequency tracking, a high $Q$ has the effect of reducing $D R$ and it has no contribution to $\delta m$. A high $Q$ reduces DR because it implies a higher thermomechanical amplitude and the appearance of nonlinear effects at lower amplitudes. In $\delta m$, the effect of better frequency tracking provided by a high $Q$ is canceled by the consequently lower DR.

In summary, we have shown that nanowire resonators with common physical characteristics can provide a broad DR: the presented measurements reach up to $90 \mathrm{~dB}$, and the theoretical analysis allows expectation of values up to the $100 \mathrm{~dB}$ level, which compares to the highest reported for any other sort of nanomechanical resonator. The presented analysis provides a foundation for the suitability of nanowire resonators as building blocks for NEMS with high performance linear operation and specifically for mass sensing applications.

\section{ASSOCIATED CONTENT}

\section{Supporting Information}

The Supporting Information is available free of charge at https://pubs.acs.org/doi/10.1021/acs.nanolett.1c02056.

Detailed theoretical description of the equations of motion of SC and DC flexural beam resonators and their nonlinear frequency response, onset of nonlinearity, and derivation of the expressions for dynamic range and fundamental mass detection limit for hexagonal, cylindrical, and rectangular beams, including the effect of tapering in SC beams; details of the Si nanowire growth and characterization; details of the transduction 
mechanism involved in the DR measurements regarding the experimental setup, the transduction sensitivity, and its linear range $(\mathrm{PDF})$

\section{AUTHOR INFORMATION}

\section{Corresponding Author}

Álvaro San Paulo - Instituto de Micro y Nanotecnología, IMN-CNM, CSIC (CEI UAM+CSIC), 28760 Tres Cantos, Madrid, Spain; $\odot$ orcid.org/0000-0001-9325-8892;

Phone: ++34.918.060.709; Email: alvaro.sanpaulo@csic.es

\section{Authors}

Juan Molina - Instituto de Micro y Nanotecnología, IMNCNM, CSIC (CEI UAM+CSIC), 28760 Tres Cantos, Madrid, Spain

Javier E. Escobar - Instituto de Micro y Nanotecnología, IMN-CNM, CSIC (CEI UAM+CSIC), 28760 Tres Cantos, Madrid, Spain

Daniel Ramos - Instituto de Micro y Nanotecnología, IMNCNM, CSIC (CEI UAM+CSIC), 28760 Tres Cantos, Madrid, Spain; ๑ orcid.org/0000-0003-2677-4058

Eduardo Gil-Santos - Instituto de Micro y Nanotecnología, IMN-CNM, CSIC (CEI UAM+CSIC), 28760 Tres Cantos, Madrid, Spain

José J. Ruz - Instituto de Micro y Nanotecnología, IMNCNM, CSIC (CEI UAM+CSIC), 28760 Tres Cantos, Madrid, Spain

Javier Tamayo - Instituto de Micro y Nanotecnología, IMNCNM, CSIC (CEI UAM+CSIC), 28760 Tres Cantos, Madrid, Spain

Montserrat Calleja - Instituto de Micro y Nanotecnología, IMN-CNM, CSIC (CEI UAM+CSIC), 28760 Tres Cantos, Madrid, Spain; $\odot$ orcid.org/0000-0003-2414-5725

Complete contact information is available at:

https://pubs.acs.org/10.1021/acs.nanolett.1c02056

\section{Notes}

The authors declare no competing financial interest.

\section{ACKNOWLEDGMENTS}

This work was supported by the ERC CoG Grant 681275 "LIQUIDMASS" and by the Spanish Science, Innovation and Universities Ministry through Projects "EXOFLUX" (PGC2018-101762-B-I00) and "MOMPs" (TEC2017-89765R). E.G.-S. acknowledges financial support by Fundación General CSIC through the ComFuturo program. We acknowledge the service from the Micro and Nanofabrication Laboratory at IMN-CNM, funded by the Comunidad de Madrid (Project S2018/NMT-4291 TEC2SPACE) and by MINECO (Project CSIC13-4E-1794 with support from FEDER, FSE).

\section{REFERENCES}

(1) Ramos, D.; Gil-Santos, E.; Malvar, O.; Llorens, J. M.; Pini, V.; Paulo, A. S.; Calleja, M.; Tamayo, J. Silicon Nanowires: Where Mechanics and Optics Meet at the Nanoscale. Sci. Rep. 2013, 3 (1), 3445.

(2) Gloppe, A.; Verlot, P.; Dupont-Ferrier, E.; Siria, A.; Poncharal, P.; Bachelier, G.; Vincent, P.; Arcizet, O. Bidimensional NanoOptomechanics and Topological Backaction in a Non-Conservative Radiation Force Field. Nat. Nanotechnol. 2014, 9 (11), 920-926.

(3) Gil-Santos, E.; Ramos, D.; Martínez, J.; Fernández-Regúlez, M.; García, R.; San Paulo, Á.; Calleja, M.; Tamayo, J. Nanomechanical
Mass Sensing and Stiffness Spectrometry Based on Two-Dimensional Vibrations of Resonant Nanowires. Nat. Nanotechnol. 2010, 5 (9), 641-645.

(4) Mercier de Lépinay, L.; Pigeau, B.; Besga, B.; Arcizet, O. Eigenmode Orthogonality Breaking and Anomalous Dynamics in Multimode Nano-Optomechanical Systems under Non-Reciprocal Coupling. Nat. Commun. 2018, 9 (1), 1401.

(5) Braakman, F. R.; Rossi, N.; Tütüncüoglu, G.; Morral, A. F. i; Poggio, M. Coherent Two-Mode Dynamics of a Nanowire Force Sensor. Phys. Rev. Appl. 2018, 9 (5), 054045.

(6) Cadeddu, D.; Braakman, F. R.; Tütüncüoglu, G.; Matteini, F.; Rüffer, D.; Fontcuberta i Morral, A.; Poggio, M. Time-Resolved Nonlinear Coupling between Orthogonal Flexural Modes of a Pristine GaAs Nanowire. Nano Lett. 2016, 16 (2), 926-931.

(7) Foster, A. P.; Maguire, J. K.; Bradley, J. P.; Lyons, T. P.; Krysa, A. B.; Fox, A. M.; Skolnick, M. S.; Wilson, L. R. Tuning Nonlinear Mechanical Mode Coupling in GaAs Nanowires Using Cross-Section Morphology Control. Nano Lett. 2016, 16 (12), 7414-7420.

(8) Nichol, J. M.; Hemesath, E. R.; Lauhon, L. J.; Budakian, R. Displacement Detection of Silicon Nanowires by PolarizationEnhanced Fiber-Optic Interferometry. Appl. Phys. Lett. 2008, 93 (19), 193110.

(9) Sanii, B.; Ashby, P. D. High Sensitivity Deflection Detection of Nanowires. Phys. Rev. Lett. 2010, 104 (14), 147203.

(10) Molina, J.; Ramos, D.; Gil-Santos, E.; Escobar, J. E.; Ruz, J. J.; Tamayo, J.; San Paulo, Á.; Calleja, M. Optical Transduction for Vertical Nanowire Resonators. Nano Lett. 2020, 20 (4), 2359-2369.

(11) Rose, W.; Haas, H.; Chen, A. Q.; Jeon, N.; Lauhon, L. J.; Cory, D. G.; Budakian, R. High-Resolution Nanoscale Solid-State Nuclear Magnetic Resonance Spectroscopy. Phys. Rev. X 2018, 8 (1), 011030.

(12) Braakman, F. R.; Poggio, M. Force Sensing with Nanowire Cantilevers. Nanotechnology 2019, 30 (33), 332001.

(13) Rossi, N.; Gross, B.; Dirnberger, F.; Bougeard, D.; Poggio, M. Magnetic Force Sensing Using a Self-Assembled Nanowire. Nano Lett. 2019, 19 (2), 930-936.

(14) Fogliano, F.; Besga, B.; Reigue, A.; Heringlake, P.; Mercier de Lépinay, L.; Vaneph, C.; Reichel, J.; Pigeau, B.; Arcizet, O. Mapping the Cavity Optomechanical Interaction with Subwavelength-Sized Ultrasensitive Nanomechanical Force Sensors. Phys. Rev. X 2021, 11 (2), 021009.

(15) de Lépinay, L. M.; Pigeau, B.; Besga, B.; Vincent, P.; Poncharal, P.; Arcizet, O. A Universal and Ultrasensitive Vectorial Nanomechanical Sensor for Imaging 2D Force Fields. Nat. Nanotechnol. 2017, 12 (2), 156-162.

(16) Rossi, N.; Braakman, F. R.; Cadeddu, D.; Vasyukov, D.; Tütüncüoglu, G.; Fontcuberta i Morral, A.; Poggio, M. Vectorial Scanning Force Microscopy Using a Nanowire Sensor. Nat. Nanotechnol. 2017, 12 (2), 150-155.

(17) Sahafi, P.; Rose, W.; Jordan, A.; Yager, B.; Piscitelli, M.; Budakian, R. Ultralow Dissipation Patterned Silicon Nanowire Arrays for Scanning Probe Microscopy. Nano Lett. 2020, 20 (1), 218-223.

(18) Tiwari, S.; Candler, R. N. Using Flexural MEMS to Study and Exploit Nonlinearities: A Review. J. Micromech. Microeng. 2019, 29 (8), 083002.

(19) Postma, H. W. Ch.; Kozinsky, I.; Husain, A.; Roukes, M. L. Dynamic Range of Nanotube- and Nanowire-Based Electromechanical Systems. Appl. Phys. Lett. 2005, 86 (22), 223105.

(20) Nichol, J. M.; Hemesath, E. R.; Lauhon, L. J.; Budakian, R. Controlling the Nonlinearity of Silicon Nanowire Resonators Using Active Feedback. Appl. Phys. Lett. 2009, 95 (12), 123116.

(21) Venstra, W. J.; Westra, H. J. R.; van der Zant, H. S. J. Mechanical Stiffening, Bistability, and Bit Operations in a Microcantilever. Appl. Phys. Lett. 2010, 97 (19), 193107.

(22) Kacem, N.; Arcamone, J.; Perez-Murano, F.; Hentz, S. Dynamic Range Enhancement of Nonlinear Nanomechanical Resonant Cantilevers for Highly Sensitive NEMS Gas/Mass Sensor Applications. J. Micromech. Microeng. 2010, 20 (4), 045023.

(23) Venstra, W. J.; Westra, H. J. R.; van der Zant, H. S. J. Stochastic Switching of Cantilever Motion. Nat. Commun. 2013, 4 (1), 2624. 
(24) Villanueva, L. G.; Karabalin, R. B.; Matheny, M. H.; Chi, D.; Sader, J. E.; Roukes, M. L. Nonlinearity in Nanomechanical Cantilevers. Phys. Rev. B: Condens. Matter Mater. Phys. 2013, 87 (2), 024304.

(25) Braakman, F. R.; Cadeddu, D.; Tütüncüoglu, G.; Matteini, F.; Rüffer, D.; Fontcuberta i Morral, A.; Poggio, M. Nonlinear Motion and Mechanical Mixing in As-Grown GaAs Nanowires. Appl. Phys. Lett. 2014, 105 (17), 173111.

(26) Lee, J.; Kaul, A. B.; Feng, P. X.-L. Carbon Nanofiber High Frequency Nanomechanical Resonators. Nanoscale 2017, 9 (33), 11864-11870.

(27) Krylyuk, S.; Davydov, A. V.; Levin, I. Tapering Control of Si Nanowires Grown from $\mathrm{SiCl}_{4}$ at Reduced Pressure. ACS Nano 2011, 5 (1), 656-664.

(28) Malvar, O.; Gil-Santos, E.; Ruz, J. J.; Ramos, D.; Pini, V.; Fernandez-Regulez, M.; Calleja, M.; Tamayo, J.; San Paulo, A. Tapered Silicon Nanowires for Enhanced Nanomechanical Sensing. Appl. Phys. Lett. 2013, 103 (3), 033101.

(29) da Silva, M. R. M. C.; Glynn, C. C. Out-of-Plane Vibrations of a Beam Including Non-Linear Inertia and Non-Linear Curvature Effects. Int. J. Non-Linear Mech. 1978, 13 (5-6), 261-271.

(30) Hamdan, M. N.; Shabaneh, N. H. ON THE LARGE AMPLITUDE FREE VIBRATIONS OF A RESTRAINED UNIFORM BEAM CARRYING AN INTERMEDIATE LUMPED MASS. J. Sound Vib. 1997, 199 (5), 711-736.

(31) Schmid, S.; Villanueva, L. G.; Roukes, M. L. Fundamentals of Nanomechanical Resonators; Springer International Publishing: Cham, Switzerland, 2016; DOI: 10.1007/978-3-319-28691-4.

(32) Ekinci, K. L.; Roukes, M. L. Nanoelectromechanical Systems. Rev. Sci. Instrum. 2005, 76 (6), 061101.

(33) Wang, Z.; Feng, P. X.-L. Dynamic Range of Atomically Thin Vibrating Nanomechanical Resonators. Appl. Phys. Lett. 2014, 104 (10), 103109.

(34) Lee, J.; Wang, Z.; He, K.; Yang, R.; Shan, J.; Feng, P. X.-L. Electrically Tunable Single- and Few-Layer $\mathrm{MoS}_{2}$ Nanoelectromechanical Systems with Broad Dynamic Range. Sci. Adv. 2018, 4 (3), No. eaao6653.

(35) San Paulo, A.; Bokor, J.; Howe, R. T.; He, R.; Yang, P.; Gao, D.; Carraro, C.; Maboudian, R. Mechanical Elasticity of Single and Double Clamped Silicon Nanobeams Fabricated by the Vapor-LiquidSolid Method. Appl. Phys. Lett. 2005, 87 (5), 053111.

(36) Zarraoa, L.; González, M. U.; Paulo, Á. S. Imaging LowDimensional Nanostructures by Very Low Voltage Scanning Electron Microscopy: Ultra-Shallow Topography and Depth-Tunable Material Contrast. Sci. Rep. 2019, 9 (1), 16263.

(37) Hauer, B. D.; Doolin, C.; Beach, K. S. D.; Davis, J. P. A General Procedure for Thermomechanical Calibration of Nano/MicroMechanical Resonators. Ann. Phys. 2013, 339, 181-207.

(38) Zhu, Y.; Xu, F.; Qin, Q.; Fung, W. Y.; Lu, W. Mechanical Properties of Vapor-Liquid-Solid Synthesized Silicon Nanowires. Nano Lett. 2009, 9 (11), 3934-3939.

(39) Kozinsky, I.; Postma, H. W. Ch.; Bargatin, I.; Roukes, M. L. Tuning Nonlinearity, Dynamic Range, and Frequency of Nanomechanical Resonators. Appl. Phys. Lett. 2006, 88 (25), 253101.

(40) Feng, X. L.; White, C. J.; Hajimiri, A.; Roukes, M. L. A SelfSustaining Ultrahigh-Frequency Nanoelectromechanical Oscillator. Nat. Nanotechnol. 2008, 3 (6), 342-346.

(41) Villanueva, L. G.; Karabalin, R. B.; Matheny, M. H.; Kenig, E.; Cross, M. C.; Roukes, M. L. A Nanoscale Parametric Feedback Oscillator. Nano Lett. 2011, 11 (11), 5054-5059.

(42) Sansa, M.; Sage, E.; Bullard, E. C.; Gély, M.; Alava, T.; Colinet, E.; Naik, A. K.; Villanueva, L. G.; Duraffourg, L.; Roukes, M. L.; Jourdan, G.; Hentz, S. Frequency Fluctuations in Silicon Nanoresonators. Nat. Nanotechnol. 2016, 11 (6), 552-558.

(43) Li, M.; Tang, H. X.; Roukes, M. L. Ultra-Sensitive NEMS-Based Cantilevers for Sensing, Scanned Probe and Very High-Frequency Applications. Nat. Nanotechnol. 2007, 2 (2), 114-120.
(44) Jensen, K.; Kim, K.; Zettl, A. An Atomic-Resolution Nanomechanical Mass Sensor. Nat. Nanotechnol. 2008, 3 (9), 533537.

(45) Malvar, O.; Ruz, J. J.; Kosaka, P. M.; Domínguez, C. M.; GilSantos, E.; Calleja, M.; Tamayo, J. Mass and Stiffness Spectrometry of Nanoparticles and Whole Intact Bacteria by Multimode Nanomechanical Resonators. Nat. Commun. 2016, 7 (1), 13452.

(46) Vidal-Álvarez, G.; Agustí, J.; Torres, F.; Abadal, G.; Barniol, N.; Llobet, J;; Sansa, M.; Fernández-Regúlez, M.; Pérez-Murano, F.; San Paulo, A.; Gottlieb, O. Top-down Silicon Microcantilever with Coupled Bottom-up Silicon Nanowire for Enhanced Mass Resolution. Nanotechnology 2015, 26 (14), 145502.

(47) Ekinci, K. L.; Yang, Y. T.; Roukes, M. L. Ultimate Limits to Inertial Mass Sensing Based upon Nanoelectromechanical Systems. J. Appl. Phys. 2004, 95 (5), 2682-2689.

(48) Yuksel, M.; Orhan, E.; Yanik, C.; Ari, A. B.; Demir, A.; Hanay, M. S. Nonlinear Nanomechanical Mass Spectrometry at the SingleNanoparticle Level. Nano Lett. 2019, 19 (6), 3583-3589. 